\title{
Energy, linear momentum, and angular momentum exchange between an electromagnetic wave- packet and a small particle
}

\section{Masud Mansuripur}

Masud Mansuripur, "Energy, linear momentum, and angular momentum exchange between an electromagnetic wave-packet and a small particle," Proc. SPIE 10712, Optical Manipulation Conference, 107121E (24 April 2018); doi: $10.1117 / 12.2317336$

SPIE. Event: SPIE Structured Light, 2018, Yokohama, Japan 


\title{
Energy, linear momentum, and angular momentum exchange between an electromagnetic wave-packet and a small particle
}

\author{
Masud Mansuripur \\ College of Optical Sciences, The University of Arizona, Tucson
}

\begin{abstract}
Invoking Maxwell's classical electrodynamics in conjunction with expressions for the electromagnetic (EM) energy, momentum, force, and torque, we use a few simple examples to demonstrate the nature of linear and angular momentum exchange between a wave-packet and a small spherical particle. The linear and angular momenta of the EM field, when absorbed by the particle, will be seen to elicit different responses from the particle.
\end{abstract}

1. Introduction. The present paper aims to illustrate the mechanisms of exchange of energy as well as linear and angular momenta between an electromagnetic (EM) wave-packet propagating in free space and a small (spherical) particle that acquires an induced polarization upon encountering the wave-packet; see Fig.1. The packet will continue to propagate beyond the particle, with its energy and momenta intact. The particle, while excited, radiates an EM wave that carries energy and angular momentum - but no linear momentum. At the same time, the particle may absorb energy as well as linear and angular momenta from the incident packet. If, in advance of the packet's arrival, the particle happens to be in an excited state (e.g., pumped gain medium), its stored internal energy will be released into the surrounding environment. Many interesting phenomena observed during radiation absorption and emission processes result from interference between the wavepacket and the EM field that has been radiated into the particle's surrounding space. We examine a few cases of practical interest.

Fig.1. (a) A wave-packet propagating in free space arrives at a small spherical particle of radius $R$ and dielectric susceptibility $\varepsilon_{0} \chi(\omega)$. (b) The particle responds to the wave-packet's $E$-field, acquiring an induced polarization $\boldsymbol{P}(t)$ for the duration of the packet. (c) The wave-packet continues on its journey, unhindered, while the dipolar radiation expands in the space surrounding the particle. Interference between the wavepacket and the (expanding) radiated field accounts for a substantial contribution to the EM energy, linear momentum, and angular momentum of the overall EM field.

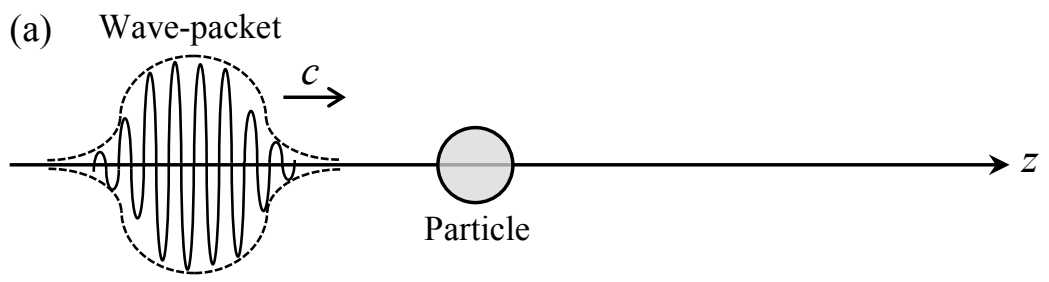

(b)
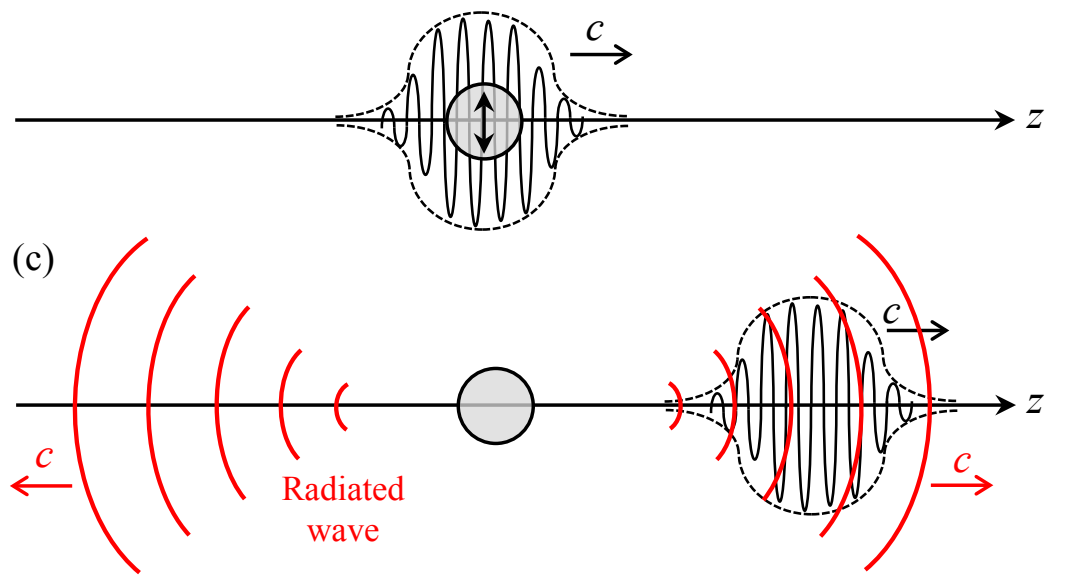

2. Formalism. The electric and magnetic fields of an infinitely-wide, uniformly-polarized, finite duration wave-packet propagating in free space along the $z$-axis may be written as superpositions of plane-waves having $k$-vectors $\left(k_{x}, k_{y}, k_{z}\right)=(0,0, \omega / c)$ and $E$-field amplitudes $\overrightarrow{\mathbb{E}}(\omega)=\mathbb{E}_{x}(\omega) \widehat{\boldsymbol{x}}+\mathbb{E}_{y}(\omega) \hat{\boldsymbol{y}}$, namely,

$$
\begin{gathered}
\boldsymbol{E}(\boldsymbol{r}, t)=\frac{1}{2 \pi} \int_{-\infty}^{\infty} \overrightarrow{\mathbb{E}}(\omega) \exp [\mathrm{i}(\omega / c)(z-c t)] \mathrm{d} \omega, \\
\boldsymbol{H}(\boldsymbol{r}, t)=\frac{1}{2 \pi z_{0}} \int_{-\infty}^{\infty} \hat{\boldsymbol{z}} \times \overrightarrow{\mathbb{E}}(\omega) \exp [\mathrm{i}(\omega / c)(z-c t)] \mathrm{d} \omega .
\end{gathered}
$$


In the above equations, $c=1 / \sqrt{\mu_{0} \varepsilon_{0}}$ is the speed of light in vacuum, and $Z_{0}=\sqrt{\mu_{0} / \varepsilon_{0}}$ is the impedance of free space $[1,2]$. The energy and linear momentum content of the packet (per unit crosssectional area) are readily found to be

$$
\begin{gathered}
\mathcal{E}=1 / 2 \varepsilon_{0} \int_{-\infty}^{\infty} \boldsymbol{E}(\boldsymbol{r}, t) \cdot \boldsymbol{E}(\boldsymbol{r}, t) \mathrm{d} z+1 / 2 \mu_{0} \int_{-\infty}^{\infty} \boldsymbol{H}(\boldsymbol{r}, t) \cdot \boldsymbol{H}(\boldsymbol{r}, t) \mathrm{d} z=\frac{1}{2 \pi z_{0}} \int_{-\infty}^{\infty} \overrightarrow{\mathbb{E}}(\omega) \cdot \overrightarrow{\mathbb{E}}^{*}(\omega) \mathrm{d} \omega . \\
\boldsymbol{p}=\int_{-\infty}^{\infty}\left[\boldsymbol{E}(\boldsymbol{r}, t) \times \boldsymbol{H}(\boldsymbol{r}, t) / c^{2}\right] \mathrm{d} z=\left(\varepsilon_{0} / 2 \pi\right) \hat{\mathbf{z}} \int_{-\infty}^{\infty} \overrightarrow{\mathbb{E}}(\omega) \cdot \overrightarrow{\mathbb{E}}^{*}(\omega) \mathrm{d} \omega=(\varepsilon / c) \hat{\mathbf{z}} .
\end{gathered}
$$

Computing the angular momentum [3-6] of the wave-packet requires a different field profile, as the uniform cross-section of the infinitely-wide packet defined by Eq.(1) is not suited for angular momentum calculations $[7,8]$. For this we must use the following finite-duration and finite cross-section wave-packet:

$$
\begin{gathered}
\boldsymbol{E}(\boldsymbol{r}, t)=\frac{1}{(2 \pi)^{3}} \iiint_{-\infty}^{\infty} \overrightarrow{\mathbb{E}}(\boldsymbol{k}) \exp [\mathrm{i}(\boldsymbol{k} \cdot \boldsymbol{r}-\omega t)] \mathrm{d} k_{x} \mathrm{~d} k_{y} \mathrm{~d} k_{z}, \\
\boldsymbol{H}(\boldsymbol{r}, t)=\frac{1}{(2 \pi)^{3} Z_{0}} \iiint_{-\infty}^{\infty}(c \boldsymbol{k} / \omega) \times \overrightarrow{\mathbb{E}}(\boldsymbol{k}) \exp [\mathrm{i}(\boldsymbol{k} \cdot \boldsymbol{r}-\omega t)] \mathrm{d} k_{x} \mathrm{~d} k_{y} \mathrm{~d} k_{z} .
\end{gathered}
$$

The frequency $\omega$ appearing in Eq.(4) is no longer an independent variable, but tied to the $k$-vector as

$$
\omega=c k_{z} \sqrt{1+\left(k_{x} / k_{z}\right)^{2}+\left(k_{y} / k_{z}\right)^{2}} .
$$

The angular momentum of the wave-packet defined by Eq.(4) will now be given in its entirety by

$$
\begin{aligned}
\mathcal{L} & =\int \boldsymbol{r} \times\left[\boldsymbol{E}(\boldsymbol{r}, t) \times \boldsymbol{H}(\boldsymbol{r}, t) / c^{2}\right] \mathrm{d} x \mathrm{~d} y \mathrm{~d} z \\
& =\frac{\mathrm{i} \varepsilon_{0}}{(2 \pi)^{3}} \iiint_{-\infty}^{\infty} \omega^{-1}\left\{\left[\left(\overrightarrow{\mathbb{E}} \cdot \partial_{k_{x}} \overrightarrow{\mathbb{E}}^{*}\right) \widehat{\boldsymbol{x}}+\left(\overrightarrow{\mathbb{E}} \cdot \partial_{k_{y}} \overrightarrow{\mathbb{E}}^{*}\right) \widehat{\boldsymbol{y}}+\left(\overrightarrow{\mathbb{E}} \cdot \partial_{k_{z}} \overrightarrow{\mathbb{E}}^{*}\right) \hat{\boldsymbol{z}}\right] \times \boldsymbol{k}+\left(\overrightarrow{\mathbb{E}}^{*} \times \overrightarrow{\mathbb{E}}\right)\right\} \mathrm{d} k_{x} \mathrm{~d} k_{y} \mathrm{~d} k_{z} .
\end{aligned}
$$

In the above equation, the second term of the integrand, namely, $\omega^{-1} \overrightarrow{\mathbb{E}}^{*} \overrightarrow{\times \mathbb{E}}$, corresponds to spin, while the remaining term represents the orbital and extrinsic angular momenta of the wave-packet [7-11].

Calculations pertaining to the dipolar field radiated by the particle-as well as those arising from interference between the radiated field and the undisturbed wave-packet-are substantially more complicated; results of these calculations (along with detailed analyses) will be reported in a longer version of the present paper. Here we limit our discussion to procedural issues along with a general description of the problem under investigation, followed by a summary of the results, which is relegated to the next section. As a procedural matter, note that, if the wave-packet's $E$ and $H$ fields are denoted by the superscript (wp), and those of the dipole radiation by the superscript (dr), then the total linear momentum of the EM field at $t=t_{0}$ may be expressed as

$$
\begin{aligned}
\boldsymbol{p}^{(\text {total })}\left(t_{0}\right) & =\frac{1}{c^{2}} \iiint_{-\infty}^{\infty}\left[\boldsymbol{E}^{(\mathrm{wp})}\left(\boldsymbol{r}, t_{0}\right)+\boldsymbol{E}^{(\mathrm{dr})}\left(\boldsymbol{r}, t_{0}\right)\right] \times\left[\boldsymbol{H}^{(\mathrm{wp})}\left(\boldsymbol{r}, t_{0}\right)+\boldsymbol{H}^{(\mathrm{dr})}\left(\boldsymbol{r}, t_{0}\right)\right] \mathrm{d} x \mathrm{~d} y \mathrm{~d} z \\
& =\boldsymbol{p}^{(\mathrm{wp})}\left(t_{0}\right)+\boldsymbol{p}^{(\mathrm{dr})}\left(t_{0}\right)+\frac{1}{c^{2}} \iiint_{-\infty}^{\infty}\left[\boldsymbol{E}^{(\mathrm{wp})} \times \boldsymbol{H}^{(\mathrm{dr})}+\boldsymbol{E}^{(\mathrm{dr})} \times \boldsymbol{H}^{(\mathrm{wp})}\right] \mathrm{d} x \mathrm{~d} y \mathrm{~d} z
\end{aligned}
$$

The latter integral in Eq.(6) represents the contribution to total EM momentum of the cross-terms produced by interference between the wave-packet and the dipole radiation. Similar expressions can be constructed for the contributions of cross-terms to total EM energy and angular momentum of the system.

We mention in passing that the dielectric susceptibility $\chi(\omega)$ of a small spherical particle of volume $v=(4 \pi / 3) R^{3}$, excited by an EM wave at the excitation frequency $\omega$ (corresponding to a wavelength $\lambda=2 \pi c / \omega)$, is given by the single-electron Lorentz oscillator model [1,2] having oscillator strength $f$, plasma frequency $\omega_{p}$, resonance frequency $\omega_{0}$, and damping coefficient $\gamma$, as follows [4]:

$$
\chi(\omega)=\frac{f \omega_{p}^{2}}{\omega_{0}^{2}-\omega^{2}-\mathrm{i}\left[\gamma \omega+\left(4 \pi^{2} f \omega_{p}^{2} / 3\right)\left(v / \lambda^{3}\right)\right]} .
$$

The term $\left(4 \pi^{2} f \omega_{p}^{2} / 3\right)\left(v / \lambda^{3}\right)$ appearing in the denominator of $\chi$ is contributed by radiation resistance. 
3. Results and Discussion. We now summarize the results of our calculations pertaining to the EM field radiated by the small spherical particle depicted in Fig.1 ("dipole radiation"), and also the computed energy as well as linear and angular momenta of the EM field that are contributed by the "cross-terms" arising from interference between the dipole radiation and the (unhindered) wave-packet, as the packet continues its propagation into the empty space beyond the particle.

Case 1: Transparent dielectric particle. In the case of a passive, non-absorptive dielectric particle, the EM energy of the dipole radiation is equal in magnitude and opposite in sign to the energy associated with the cross-terms in the region where the dipole radiation and the wave-packet overlap. The radiated field does not carry any linear momentum, but the cross-terms resulting from interference contribute to the overall momentum of the EM field. The EM momentum contributed by the cross-terms is equal in magnitude and opposite in sign to the mechanical momentum picked up by the particle during its encounter with the wave-packet. The transparent particle does not experience a net torque, indicating that the angular momentum content of the radiated dipolar field is cancelled out by the angular momentum contributed by the cross-terms of the interference pattern.

Case 2: Absorptive particle. Once again, the particle picks up some linear momentum from the wavepacket, but this time it could also take away some of the energy and angular momentum of the incident beam, converting the latter to mechanical angular momentum. As before, the directly radiated EM field carries some energy and angular momentum, but no linear momentum. The cross-terms arising in consequence of interference between the wave-packet and the dipole radiation contribute to the overall energy, linear momentum, and angular momentum of the EM field. However, these cross-terms do not fully cancel out the contributions of the dipolar field, because some energy now resides within the particle and, having experienced a net torque, the particle acquires some angular momentum as well. Needless to say, the particle also picks up some linear momentum, but this is similar, qualitatively speaking, to the case of a non-absorptive particle.

Case 3: Pre-excited (or gainy) particle. The susceptibility of the particle in the case of a gain medium has a negative oscillator strength $f$, which ensures that the EM energy of the dipole radiation plus the contribution to the energy by the cross-terms within the interference zone end up making a net positive contribution; this net addition to the EM energy is caused by the (stimulated) extraction of the stored energy of the pre-excited particle. The linear and angular momenta of the EM field will likewise receive contributions from the stored energy.

Our analysis of the present case sheds light on the stimulated emission process, considering that the stored energy of the particle primarily contributes to the cross-terms arising from interference between the dipole radiation and the (unhindered) wave-packet. It is also possible in this case to examine the effects of vacuum fluctuations on the emission process, thus drawing conclusions about the process of spontaneous emission. These and other related issues will be addressed in our conference presentation.

1. J. D. Jackson, Classical Electrodynamics (3 ${ }^{\text {rd }}$ edition), Wiley, New York (1999).

2. M. Mansuripur, Field, Force, Energy and Momentum in Classical Electrodynamics (revised edition), Bentham e-books, Sharjah, U.A.E. (2017).

3. H. Rubinsztein-Dunlop and A. Forbes (editors), "Roadmap on Structured Light," Journal of Optics 19, 013001,1-51 (2017).

4. M. Mansuripur, "Optical Angular Momentum in Classical Electrodynamics," Physica Scripta 92, 065501, pp1-9 (2017).

5. M. Mansuripur, "Angular momentum of circularly polarized light in dielectric media," Optics Express 13, 5315-24 (2005).

6. M. V. Berry, M. R. Jeffrey, and M. Mansuripur, "Orbital and spin angular momentum in conical diffraction," Journal of Optics A: Pure and Applied Optics 7, 685-690 (2005).

7. M. Mansuripur, "Spin and orbital angular momenta of electromagnetic waves in free space," Phys. Rev. A 84, 033838 (2011).

8. M. Mansuripur, "Energy and linear and angular momenta in simple electromagnetic systems," Optical Trapping and Optical Micromanipulation XII, edited by K. Dholakia and G. C. Spalding, Proceedings of SPIE 9548, 95480K 1:24 (2015).

9. L. Allen, S. Barnett, and M. Padgett, Optical Angular Momentum, Institute of Physics Publishing, Bristol, U.K. (2003).

10. S. Barnett, "Rotation of electromagnetic fields and the nature of optical angular momentum," J. Mod. Opt. 57, 1339 (2010).

11. L. Marrucci, E. Karimi, S. Slussarenko, B. Piccirillo, E. Santamato, E. Nagali, and F. Sciarrino, "Spin-to-orbital conversion of the angular momentum of light and its classical and quantum applications," Journal of Optics 13, 064001 (2011). 